\title{
A New Class of Cascade Orthogonal Filters based on a Special Inner Product with Application in Modeling of Dynamical Systems
}

\author{
Nikola B. Danković, Dragan S. Antić, Saša S. Nikolić, Staniša Lj. \\ Perić, Marko T. Milojković
}

University of Niš, Faculty of Electronic Engineering, Department of Control

Systems, Aleksandra Medvedeva 14, 18000 Niš, Serbia

nikola.dankovic@elfak.ni.ac.rs,dragan.antic@elfak.ni.ac.rs,

sasa.s.nikolic@elfak.ni.ac.rs, stanisa.peric@elfak.ni.ac.rs,

marko.milojkovic@elfak.ni.ac.rs

\begin{abstract}
A class of cascade filters, orthogonal with respect to a new inner product, is presented in this paper. A sequence of generalized Malmquist orthogonal rational functions is used for design of these filters. In addition, by using these functions Müntz polynomials which are orthogonal in respect to a special inner product were derived. Obtained Müntz polynomials are applied in determination of outputs of the proposed filters. Depending on whether the design of the filters is performed in the s-domain or complex z-domain, we can derive a class of analogue or digital filters, respectively. Outputs from these filters are orthogonal with respect to the two different inner products. Both classes of filters are practically realized and their application in modeling of continuous-time and discrete-time dynamical systems is given. Obtained results show that there are great agreements between the outputs of models and real dynamical systems.
\end{abstract}

Keywords: orthogonal filters; inner product; dynamical systems modeling; Malmquist functions; Müntz polynomials

\section{Introduction}

Around the middle of the last century, a new class of orthogonal rational functions were developed [1-3], and later they were used for synthesis of orthogonal filters. Also, classes of generalized Legendre polynomials and generalized orthogonal polynomials of Szegö were derived [4, 5]. Further generalization of classical orthogonal polynomials and orthogonal rational functions is given in [6,7]. Derivation of Müntz orthogonal polynomials from these functions is described, as well in [8]. An overview of the theory of such orthogonal systems and some problems in applications of orthogonal polynomials are given in [9]. A few years later, the paper [9] was translated to English [10]. 
A method for obtaining a sequence of orthogonal rational functions is given in [11-14]. The method is based on the pole-zero and zero-pole mapping by using symmetric transformation. In this way obtained rational functions were used to design of new classes of orthogonal filters, quazi-orthogonal filters [15], almost orthogonal filters [16-21] and finally, generalized orthogonal filters with complex poles [22]. Using the same method, new classes of Malmquist orthogonal functions can also be obtained. These classes involve already known classes of Malmquist rational functions [23]. The method for obtaining Müntz orthogonal polynomials from sequence of orthogonal Malmquist functions is presented in [13, 14].

In this paper, generalized Malmquist functions are used for derivation of Müntz orthogonal polynomials (associated Müntz polynomials). By using these polynomials, a new class of filters, orthogonal with respect to a special inner product, is designed. Orthogonal rational function was derived by using the special symmetric transformation for pole-zero mapping and vice versa. Rational functions are used for structure design of filters, and appropriate Müntz polynomials are used to determine the outputs of these filters. Two subclasses of these filters are designed: subclass of analogue filters and subclass of digital filters. Practical realization of these filters was performed and their applications in modeling continual and discrete systems are given.

\section{The Sequence of Orthogonal Rational Functions and Associated Müntz Orthogonal Polynomials}

Let us introduce the sequence of rational functions:

$$
W_{n}(s)=\frac{\prod_{k=0}^{n-1}\left(s-\alpha_{k}^{*}\right)}{\prod_{k=0}^{n}\left(s-\alpha_{k}\right)}, \quad n=1,2,3, \ldots
$$

where the zeroes $\alpha_{k}^{*}$ are obtained by mapping the poles $\alpha_{k}$, and the poles $\alpha_{k}$ are obtained by mapping the zeroes $\alpha_{k}^{*}$, using the following symmetric transformation:

$\alpha_{k}^{*}=f\left(\alpha_{k}\right), \alpha_{k}=f\left(\alpha_{k}^{*}\right)$.

Substituting (2) in (1), transformed sequence is obtained: 


$$
\overline{W_{n}(s)}=\frac{\prod_{k=0}^{n-1}\left(s-\alpha_{k}\right)}{\prod_{k=0}^{n}\left(s-\alpha_{k}^{*}\right)} .
$$

As we can see, the zeroes of the transformed sequence are equal to the poles of $W_{n}(s)$ and vice versa.

Now, let us consider the inner product:

$$
J_{n, m}=\left(W_{n}(s), \overline{W_{m}(s)}\right)=\frac{1}{2 \pi i} \int_{C_{P}} W_{n}(s) \overline{W_{m}(s)} d s,
$$

where $C_{p}$ involves all the poles of $W_{n}(s)$. In the cases of: $m>n$ and $m<n$, poles in the integrand (4) inside the contour $C_{p}$ are annulled with appropriate zeroes. Applying Cauchy theorem, we have $J_{n, m}=0$. In the case of $m=n$, there exists one first-order pole inside the contour $C_{p}$, so $J_{n, n}=\int_{C_{P}} W_{n}(s) W_{n}(s) d s=N_{n}{ }^{2}$. Hence, applying symmetric transformation of poles to zeroes of $W_{n}(s)$, we obtain the orthogonal sequence of the rational functions.

Let us apply symmetric transformation which maps the zeroes to poles and the poles to zeroes of $W_{n}(s)$ in the following way: $\alpha_{k}^{*}=\frac{b}{\overline{\alpha_{k}}}$, i.e., $\alpha_{k}=\frac{b}{\overline{\alpha_{k}^{*}}}$, to the sequence (1). In this way, we obtain two sequences orthogonal with respect to inner product (4):

$$
W_{n}(s)=\frac{\prod_{k=0}^{n-1}\left(s-\frac{b}{\overline{\alpha_{k}}}\right)}{\prod_{k=0}^{n}\left(s-\alpha_{k}\right)}, \overline{W_{m}}(s)=\frac{\prod_{k=0}^{n-1}\left(s-\alpha_{k}\right)}{\prod_{k=0}^{n}\left(s-\frac{b}{\overline{\alpha_{k}}}\right)},
$$

where $W_{n}(s)$ and $\overline{W_{n}}(s)$ are generalized Malmquist functions.

Using (4) and (5), we obtain:

$$
\left(W_{n}(s) \overline{W_{m}(s)}\right)=\frac{b^{n}}{\left(\left|\alpha_{n}\right|^{2}-b\right) \prod_{k=1}^{n-1}\left|\alpha_{k}\right|^{2}} \delta_{n, m} .
$$


One class of Müntz polynomials, orthogonal on $(0,1)$, derived from the sequence of orthogonal rational function is given in $[8,11]$. In the similar way, Müntz polynomials [9] can be obtained from the orthogonal sequence (5) as follows:

$$
P_{n}(x)=\frac{1}{2 \pi i} \oint_{C_{P}} W_{n}(s) x^{s} d s .
$$

Using (5) and (7), we obtain:

$$
P_{n}(x)=\sum_{k=0}^{n} A_{n, k} x^{\alpha_{k}}
$$

where: $A_{n, k}=\frac{\prod_{j=0}^{n-1}\left(\alpha_{k}-\frac{b}{\alpha_{j}}\right)}{\prod_{j=0, j \neq k}^{n}\left(\alpha_{k}-\alpha_{j}\right)}, k=0,1,2, \ldots, n$.

A new operator for product over monoms $x^{\alpha}$ and $x^{\beta}$ is defined in the following manner [9]:

$$
x^{\alpha} \square x^{\beta}=x^{\alpha \beta} .
$$

Then, we define a new product of two Müntz polynomials $P_{n}(x)=\sum_{k=0}^{n} p_{k} x^{\alpha_{k}}$ and $P_{m}(x)=\sum_{j=0}^{m} q_{j} x^{\alpha_{j}}$ in the following way:

$$
\left(P_{n} \square P_{m}\right)(x)=\sum_{k=0}^{n} \sum_{j=0}^{m} p_{k} q_{j} x^{\alpha_{k} \alpha_{j}} .
$$

On the basis of the product of polynomials defined above, the new inner product can be defined as:

$$
\left(P_{n}, P_{m}\right)_{\|}=\int_{0}^{1}\left(P_{n} \square \bar{P}_{m}\right)(x) \frac{d x}{x^{2}} .
$$

Applying this inner product to Müntz polynomials (8), we obtain:

$$
\left(P_{n}(x), P_{m}(x)\right)_{\square}=\frac{b^{n}}{\left(\left|\alpha_{n}\right|^{2}-b\right) \prod_{k=0}^{n-1}\left|\alpha_{k}\right|^{2}} \delta_{n, m} .
$$

In this way, we showed that Müntz polynomials obtained by using a class of generalized Malmquist functions are orthogonal with respect to the new inner product (12). A connection between orthogonal sequences of rational functions 
(generalized Malmquist functions) and Müntz orthogonal polynomials is established (see Eq. 6).

\section{Design of a New Class of Orthogonal Filters}

When $W_{n}(s)$ have real poles, then associated Müntz polynomials have real exponents. In this case, substituting $x=e^{t}$ into $P_{n}(x)$, we obtain the sequence of exponential functions:

$\varphi_{n}(t)=P_{n}\left(e^{t}\right)=\sum_{k=0}^{n} A_{n, k} e^{\alpha_{k} t}$.

These functions are orthogonal with respect to the new inner product:

$\left(\varphi_{n}(t), \varphi_{m}(t)\right)_{\sqcap}=\int_{0}^{\infty} \varphi_{n}(t) \square \varphi_{m}(t) e^{-t} d t$,

where: $\varphi_{n}(t) \square \varphi_{m}(t)=\sum_{k=0}^{n} \sum_{j=0}^{m} A_{n, k} A_{m, j} e^{\alpha_{k} \alpha_{j} t}$.

The sequence of orthogonal rational functions (5) provides for the design of a new class of cascade orthogonal filters. Let us suppose that these functions have real poles. Now, we can design a cascade filter with real poles shown in Figure 1.

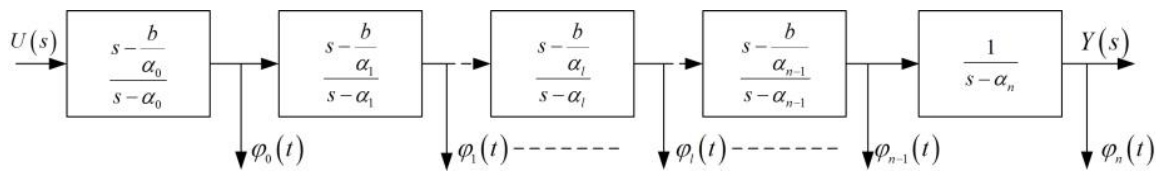

Figure 1

Block diagram of proposed orthogonal filter

Outputs from the filter in the time domain are:

$\varphi_{l}(t)=L^{-1}\left\{W_{l}(s)\right\}=\frac{1}{2 \pi i} \int_{C_{p}} W_{l}(s) e^{s t} d s$,

where: $W_{l}(s)=\prod_{k=0}^{l} \frac{s-\frac{b}{\alpha_{k}}}{s-\alpha_{k}}, l=0,1,2, \ldots, n$.

Therefore, comparing (7), (8), and (15), we can notice that filter outputs are determined when we introduce substitution $x=e^{t}$ in Müntz polynomials (8), 
obtained using the sequence of rational functions $W_{l}(s)$. Thereby, the contour $C_{p}$ involves all poles of $W_{l}(s)$, and all zeroes of $W_{l}(s)$ lie outside this contour.

Outputs $\varphi_{l}(t)$ are orthogonal in the time domain on the interval $(0, \infty)$ with respect to the new inner product (14). The diagram in Figure 1 is used for design of two new classes of orthogonal filters: analogue and digital version. The class of analogue orthogonal filters is obtained when we introduce $s$ as an operator for differentiating. These filters are orthogonal in the complex domain on the contour which involves all poles of the filter. In the time domain, the filter outputs $\varphi_{l}(t)$ are orthogonal with respect to the inner product (14).

Digital orthogonal filters are obtained after moving in $z$-domain using the transformation $s=\frac{1}{T} \ln z$, where $z$ presents the operator of prediction for one sample period in the time domain. These filters are also orthogonal in the complex domain, while outputs in the time domain are orthogonal in the classical sense.

\section{Practical Realization of the New Orthogonal Filters}

The obtained filter block scheme given in Figure 1 is suitable for practical implementation. For the purpose of analogue filter realization, we will write the transfer function in the following form:

$W_{n}(s)=\frac{\prod_{k=0}^{n-1}\left(s+\alpha_{k}^{*}\right)}{\prod_{k=0}^{n}\left(s+\alpha_{k}\right)}=\frac{1}{s+\alpha_{0}} \prod_{k=1}^{n} \frac{s+\alpha_{k-1}^{*}}{s+\alpha_{k}}, \alpha_{k}^{*}=\frac{b}{\alpha_{k}}, \alpha_{k} \in R, \quad \alpha_{k} \geq 0$.

In such a way the modified filter is orthogonal both in the complex and time domain. We have practically realized proposed filter in our Laboratory for modeling, simulation, and control systems and it is shown in Figure 2. The setup includes a printed circuit board with the realized new type of orthogonal filter, a microprocessor unit, an acquisition unit, and power supply. The realized filter has real and adjustable poles. 


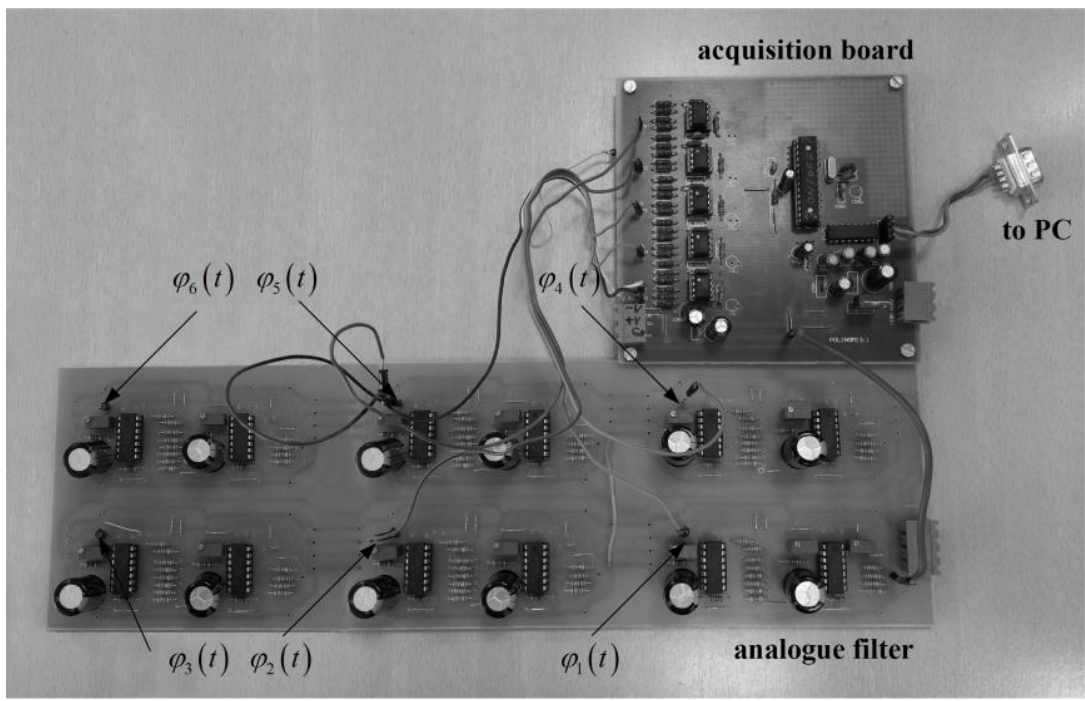

Figure 2

The analogue orthogonal filter, a printed circuit board

For the illustrative purpose, a sequence of functions on the outputs of the first five cascades of the proposed analogue filter (16) for the following values $b=-1, \alpha_{0}=1, \alpha_{1}=2, \alpha_{2}=3, \alpha_{3}=4, \alpha_{4}=5$ is obtained mathematically and given by:

$\varphi_{0}(t)=e^{-t}, \varphi_{1}(t)=3 e^{-2 t}-2 e^{-t}$,

$\varphi_{2}(t)=7 e^{-3 t}-\frac{15}{2} e^{-2 t}+\frac{3}{2} e^{-t}, \varphi_{3}(t)=\frac{65}{4} e^{-4 t}-\frac{70}{3} e^{-3 t}+\frac{35}{4} e^{-2 t}-\frac{2}{3} e^{-t}$,

$\varphi_{4}(t)=\frac{77}{2} e^{-5 t}-\frac{1105}{16} e^{-4 t}+\frac{455}{12} e^{-3 t}-\frac{105}{16} e^{-2 t}+\frac{5}{24} e^{-t}$.

The outputs from the realized filter are shown in Figure 3. Orthogonality with respect to the new inner product (14) of these outputs can be easily verified.

As we have already said, when we get into $z$-domain (i.e., the operator $s$ is substituted with $z$ ), a digital filter can be realized on the basis of the modified scheme by using the following transfer function:

$W_{n}(z)=\frac{z}{z-\alpha_{0}} \prod_{k=1}^{n} \frac{z-\alpha_{k-1}^{*}}{z-\alpha_{k}}, \alpha_{k}^{*}=\frac{b}{\alpha_{k}}, \alpha_{k} \in R$.

Practical realization of the proposed digital filter, which is also performed in our laboratory, is shown in Figure 4. It too, has real and adjustable poles. 


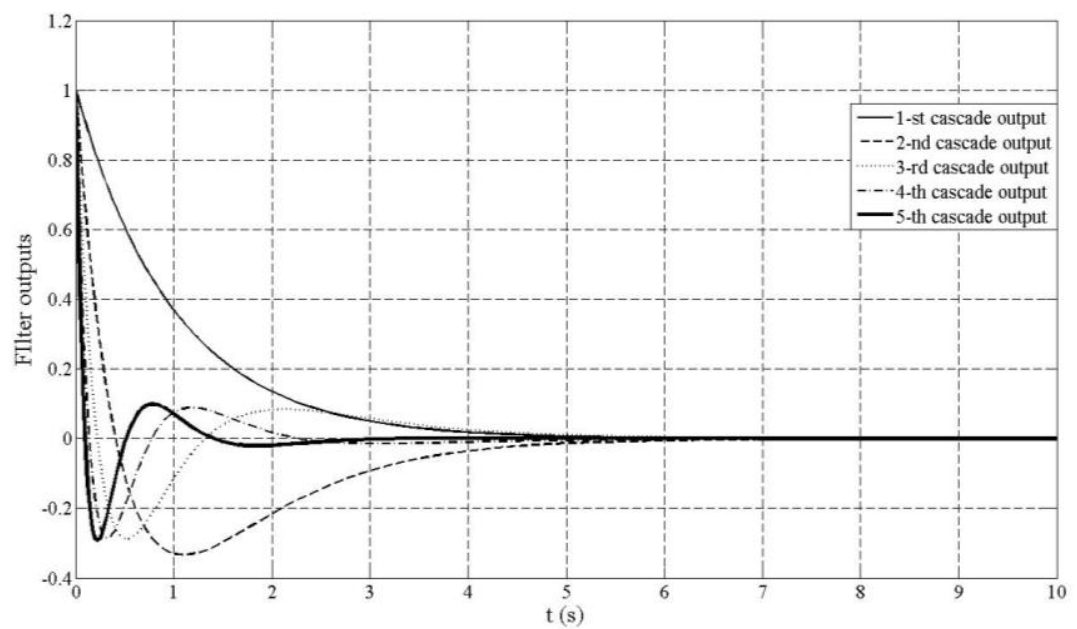

Figure 3

Outputs $\varphi_{l}(t)$ from the analogue filter, signals sensed on a printed circuit board

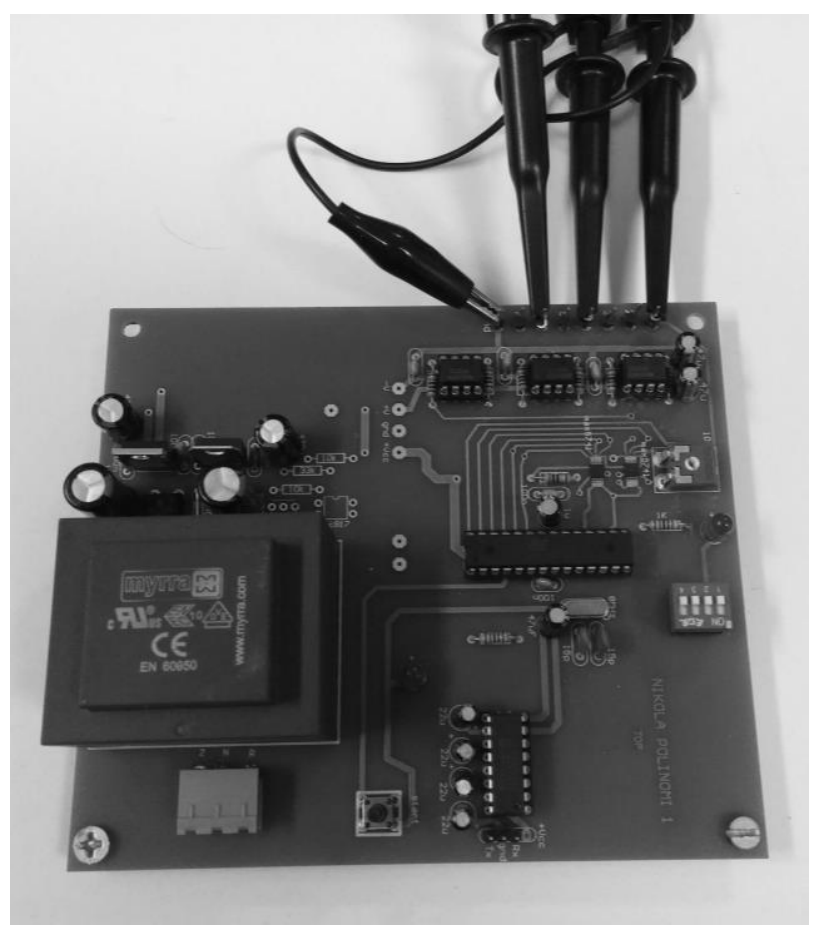

Figure 4

The digital orthogonal filter, a printed circuit board 
A sequence of functions on the outputs of cascades of the proposed digital filter (18) is obtained mathematically for the following values $b=1, \alpha_{0}=\frac{1}{2}, \alpha_{1}=\frac{1}{3}, \alpha_{2}=\frac{1}{4}, \alpha_{3}=\frac{1}{5}$, and the first few outputs are given by:

$\varphi_{0}(K)=\left(\frac{1}{2}\right)^{K}, \varphi_{1}(K)=10\left(\frac{1}{3}\right)^{K}-9\left(\frac{1}{2}\right)^{K}$,

$\varphi_{2}(K)=231\left(\frac{1}{4}\right)^{K}-320\left(\frac{1}{3}\right)^{K}+90\left(\frac{1}{2}\right)^{K}$,

$\varphi_{3}(K)=9576\left(\frac{1}{5}\right)^{K}-17325\left(\frac{1}{4}\right)^{K}+8800\left(\frac{1}{3}\right)^{K}-1050\left(\frac{1}{2}\right)^{K}$.

The same outputs are obtained by simulation in the Matlab/Simulink software package (Figure 5) and from the practically realized digital filter (Figure 6).
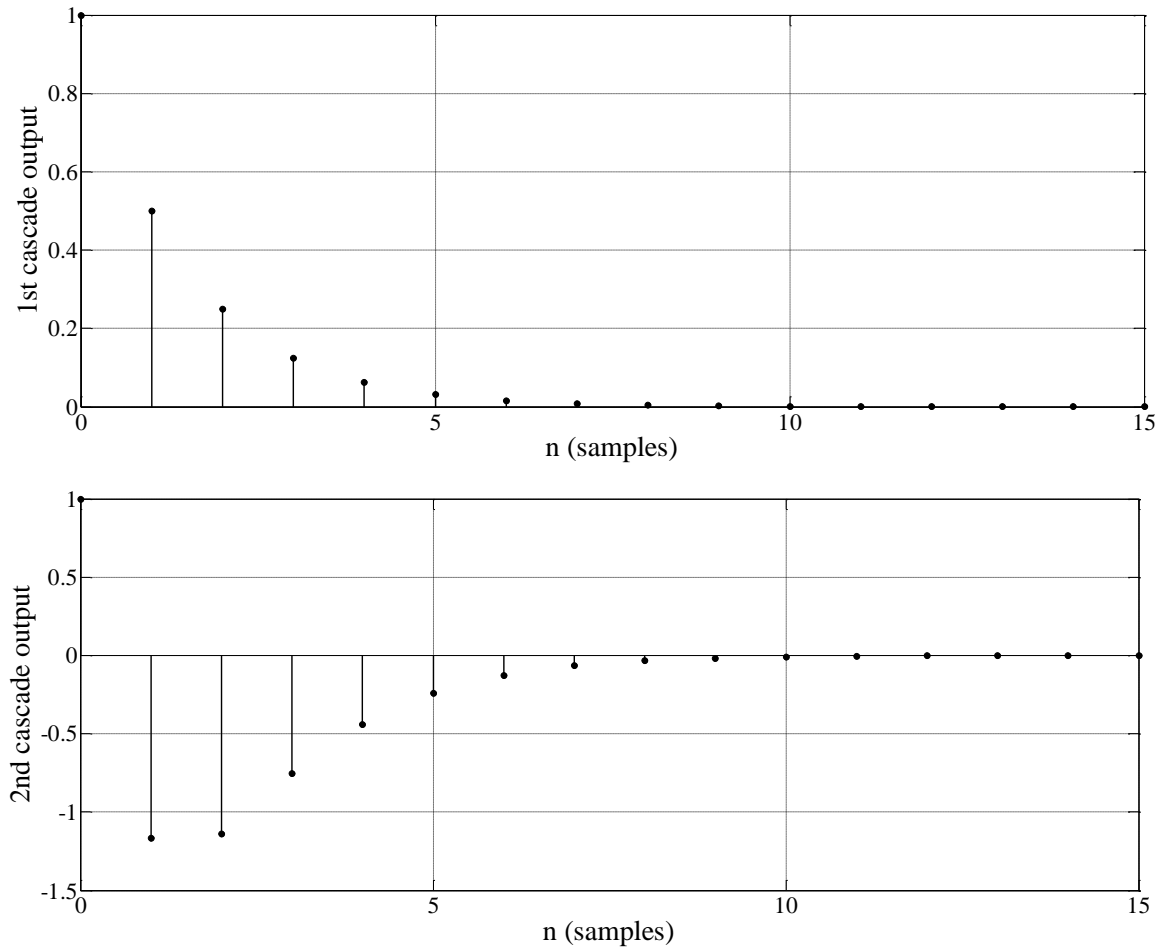

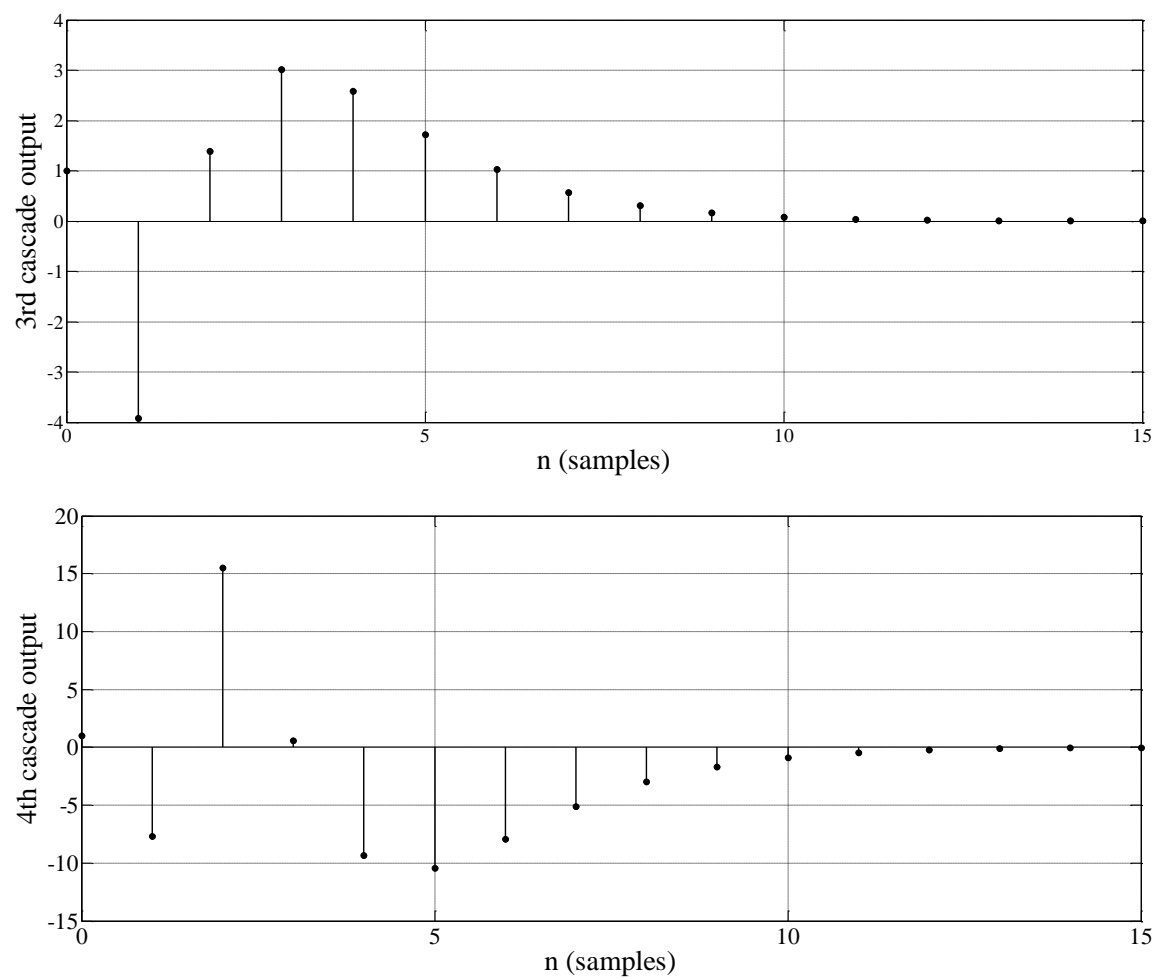

Figure 5

Outputs $\varphi_{l}(K), \quad l=0,1,2,3$ from the digital filter, signals sensed in Matlab

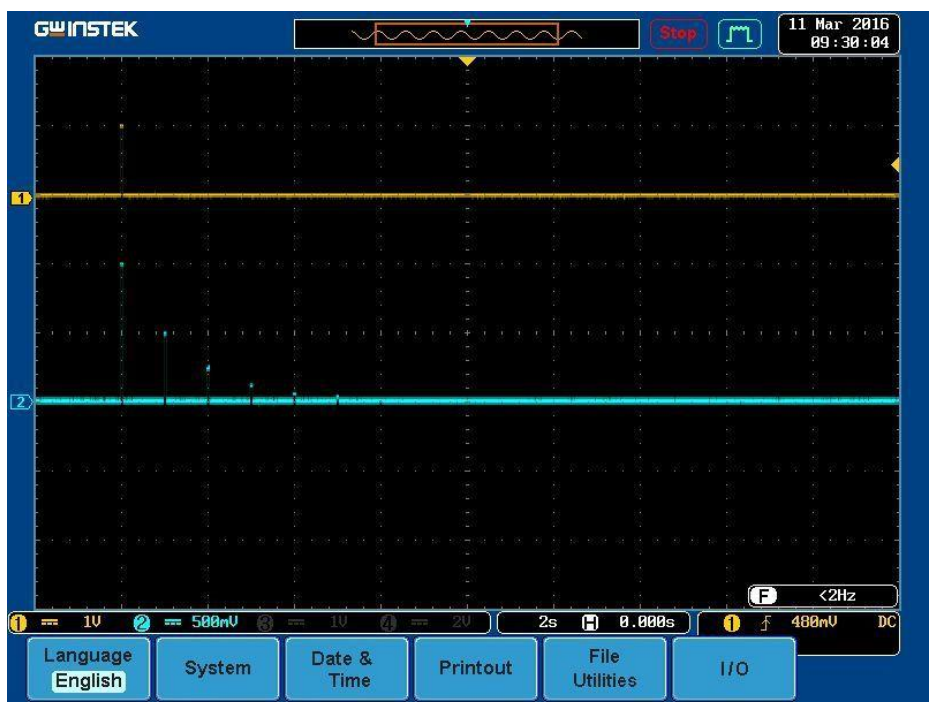



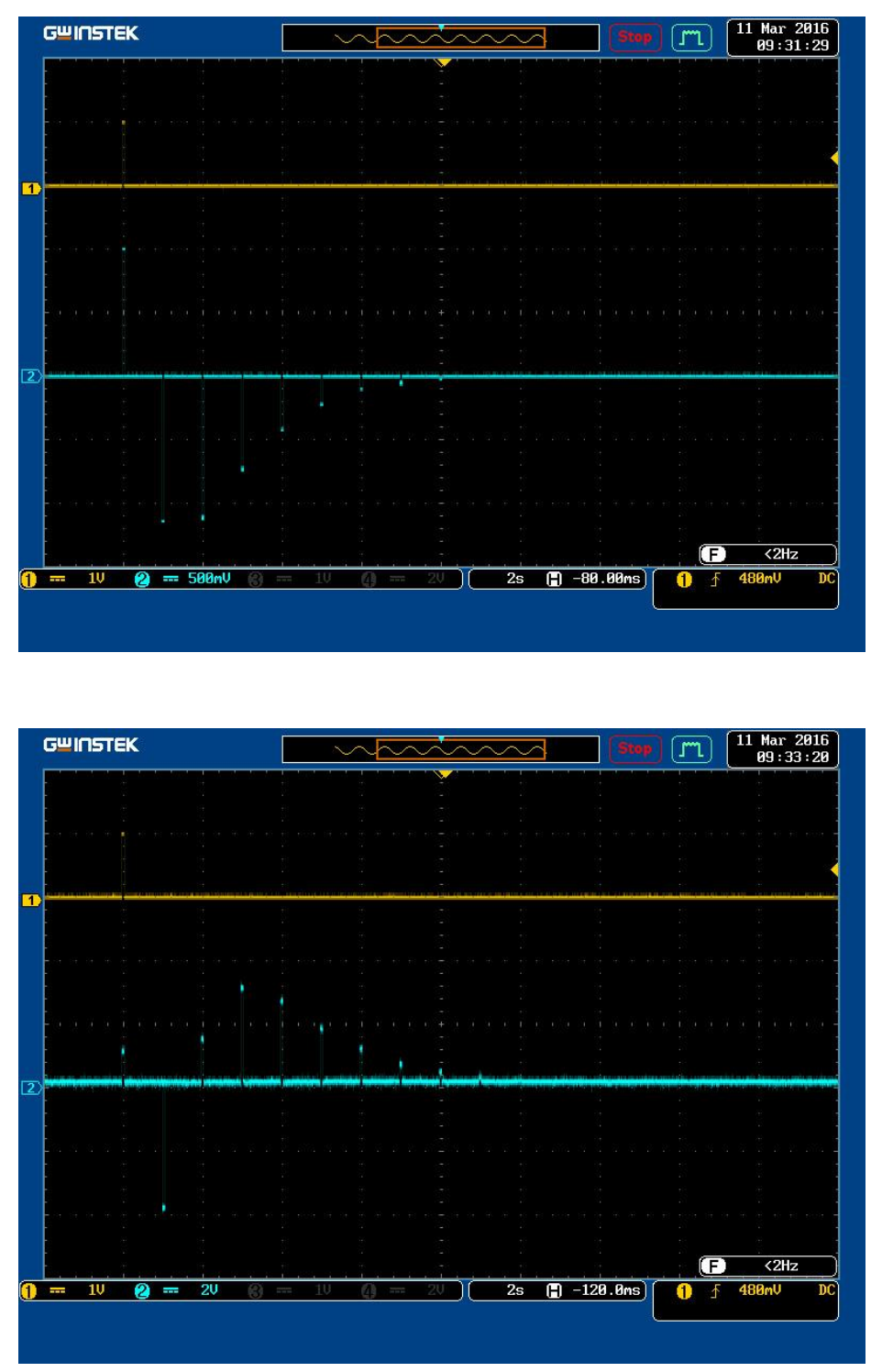


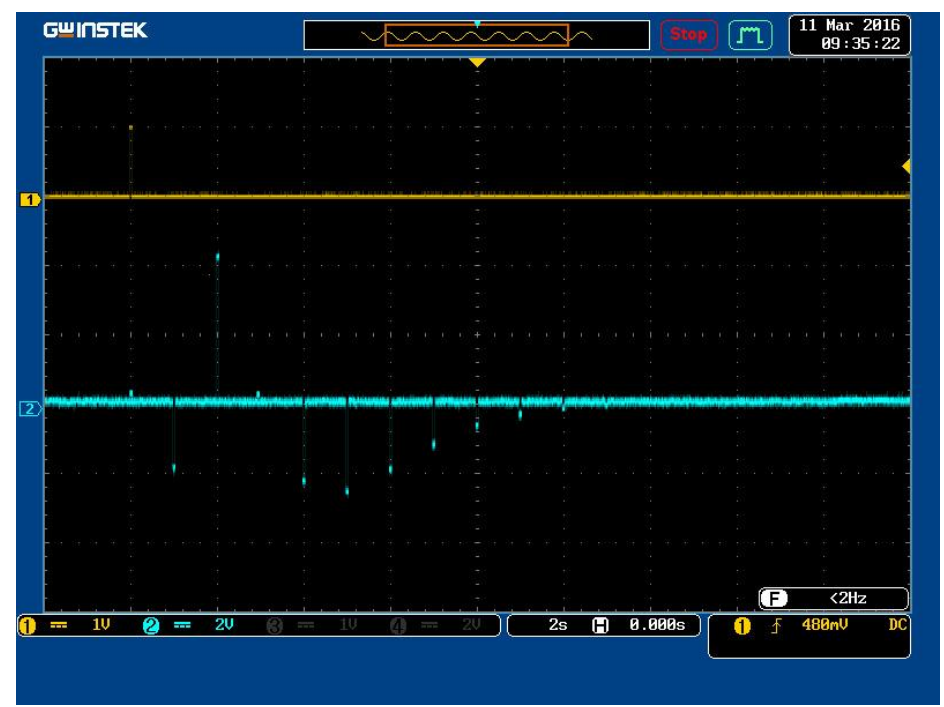

Figure 6

Outputs $\varphi_{l}(K), l=0,1,2,3$ from the digital filter, signals sensed on a printed circuit board

Channel 1 in Figure 6 represents a step input signal, while channel 2 represents an appropriate signal from the filter section. Signals were recorded by using GW INSTEC digital storage oscilloscope (series GDS-3254).

The outputs from realized filter are orthogonal with respect to the inner product (14):

$J_{n, m}=\left(\varphi_{m}(K), \varphi_{n}(K)\right)=\sum_{k=1}^{\infty} \varphi_{m}(K) \varphi_{n}(K)=N_{n}^{2} \delta_{n, m}$.

\section{Case Study-Application in Modeling Continuous and Discrete Systems}

Analogue and digital versions of the proposed orthogonal filters will be applied by modeling one continuous and one discrete system, both well known and commonly used in practice.

\subsection{Modeling of a Protector Cooling System}

The analogue version of the newly designed cascade orthogonal filter has been applied in modeling of one technological process in the tyre industry. It is a process of tyre strip cooling, more precisely protector (outer part of a tyre) cooling $[15,22,24]$. This is a complex electromechanical and thermodynamic system 
which usually consists of 5-16 cascades about $15 \mathrm{~m}$ long. There are about several hundred systems like this in the world. The model is obtained by successively modeling cascades, starting with the first one. Modeling is performed applying a genetic algorithm. We used the adjustable model shown in Figure 7. Thereby, the output from $i$-th cascade in the model is described by:

$y_{M, i}=\sum_{k=0}^{3} b_{k} \varphi_{k}(t), \quad i=1,2, \ldots, N$,

where $N$ is an order number of the system cascade, and $b_{j}$ are summation coefficients.

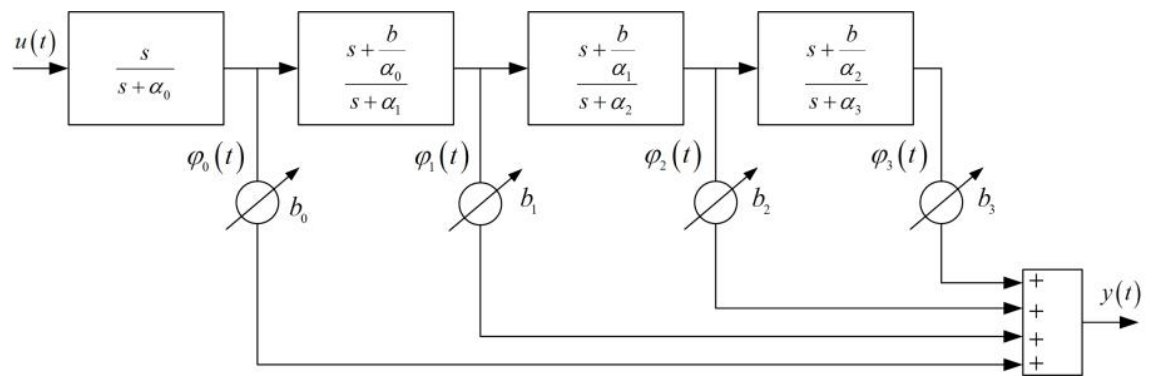

Figure 7

Block diagram of an adjustable model with the proposed orthogonal analogue filter

For determining model of the first cascade, step response is used, while for the models of other cascades, previous ones are used (for modeling $i$-th cascade, $(i-1)$ th cascade is used).

The outputs from the first cascade of the process, $y_{S}(t)$, and the model, $y_{M}(t)$, are shown in Figure 8.

Using a genetic algorithm with minimization of the mean squared error $J=\frac{1}{N} \int_{0}^{N}\left(y_{S}(t)-y_{M}(t)\right)^{2} d t$, we obtained poles of the process $\alpha_{i}$, summation coefficients $b_{i}$, and mapping parameter $b$. In our previously study, we have already used the genetic algorithm for the adjustment of parameters and minimization of criteria function $J[14,17,22]$.

The specific genetic algorithm used in experiments has the following parameters: initial population of 1000 individuals, a number of generations of 600, a stochastic uniform selection, a reproduction with ten elite individuals, and Gaussian mutation with shrinking. The used structure of chromosome was with 8 parameters coded by real numbers: $\alpha_{0}, \alpha_{1}, \alpha_{2}, \alpha_{3}, b_{0}, b_{1}, b_{2}$ and $b_{3}$. The main goal of the experiments was to obtain the best model of the unknown system in regard to the criteria function, i.e. mean squared error. 


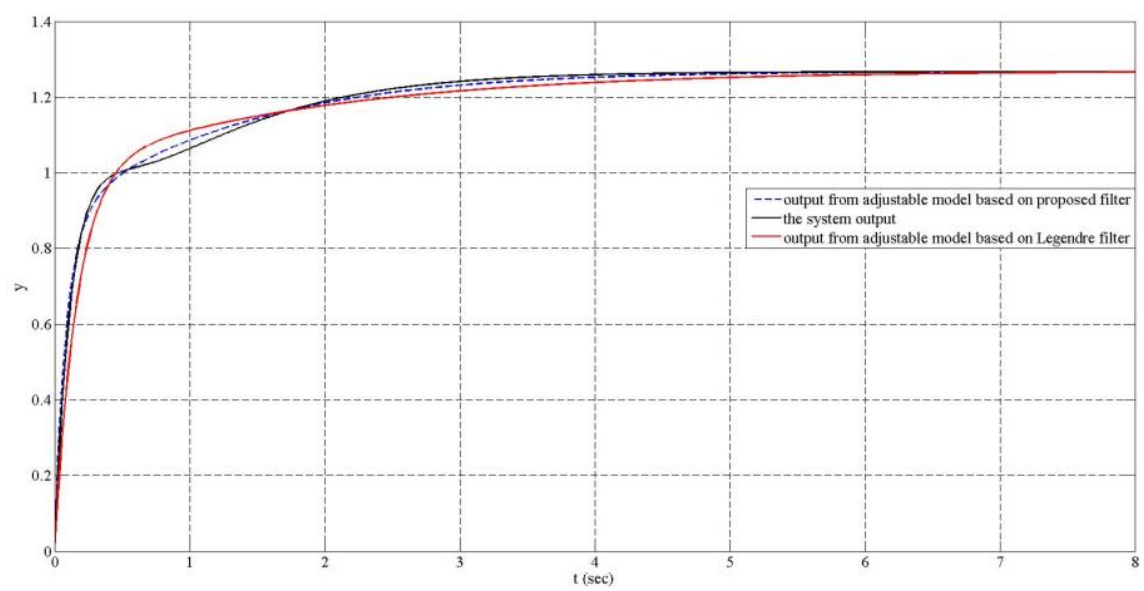

Figure 8

Outputs from the first cascade of a protector cooling system and the adjustable model

The model of the first cascade has the following form:

$W(z)=\frac{b_{0}}{s+\alpha_{0}}+\sum_{k=1}^{3} b_{k} \frac{s+\frac{b}{\alpha_{k}}}{s+\alpha_{k}}$,

where $b=0.62$ and obtained values for poles and summation coefficients are given in Table 1. The obtained value for the mean squared error is: $J_{\min }=0.77954 \cdot 10^{-3}$.

Table 1

Obtained parameters of the orthogonal analogue model

\begin{tabular}{|l|l|l|l|l|}
\hline $\mathrm{k}$ & 0 & 1 & 2 & 3 \\
\hline$\alpha_{k}$ & 1.68 & 1.21 & 4.53 & 6.53 \\
\hline$b_{k}$ & 2.08 & -0.22 & 3.35 & 3.35 \\
\hline
\end{tabular}

The transfer function of the model (22) after substituting the values from Table 1 can be rewritten in the following way:

$$
W(s)=\frac{8.57 s^{3}+51.31 s^{2}+103.12 s+76.38}{s^{4}+13.96 s^{3}+63.65 s^{2}+108.17 s+60.25} .
$$

In order to verify quality of the model based on the proposed orthogonal filter, comparison with the model based on orthogonal Legendre's filter was performed (zeroes of the filter, $\alpha_{k}^{*}$ are shifted for $l$ related to poles $\alpha_{k}$ [21, 22]). Filter shown in Figure 7 is now modified according to the mapping function $\alpha_{k}^{*}=\alpha_{k}+l$, and 
outputs from both models are given in Figure 8. The following results were obtained: $l=0.7, \alpha_{0}=5.82, \alpha_{1}=4.20, \alpha_{2}=0.54, \alpha_{3}=1.31, b_{0}=6.48, b_{1}=-0.38, b_{2}=0.18$, $b_{3}=-0.08$, and $J_{\min }=5.3457 \cdot 10^{-3}$. Hence, in this case, mean squared error is much bigger.

\subsection{Modeling of a Linear Part of DPCM System}

On the other hand, a cascade orthogonal digital filter has been applied in modeling of the linear part of differential pulse code modulation transmission system [25]. Differential Pulse Code Modulation-DPCM is a well known and commonly used technique for signal transmission in telecommunications. An estimation, i.e. a prediction of the present value of the input signal is based on knowledge of its earlier values [26, 27]. This is why one of the most important parts of every DPCM and ADPCM (Adaptive Differential Pulse Code Modulation) is a predictor (a linear part of the system). The linear part in DPCM encoder will be modeled. In the encoder, the predictor is situated in the direct branch of a positive feedback loop as opposed to the decoder [27].

For the purpose of modeling a linear part of the encoder in DPCM system (in further text DPCM linear part), we use an adjustable model shown in Figure 9, which is based on the new orthogonal digital filter. In this particular case, we use filter with six sections and it has real and adjustable poles.

The output from the orthogonal model can be calculated as:

$$
y_{M}(K)=\sum_{k=0}^{n} b_{k} \varphi_{k}(K),
$$

where $K$ represents the number of samples.

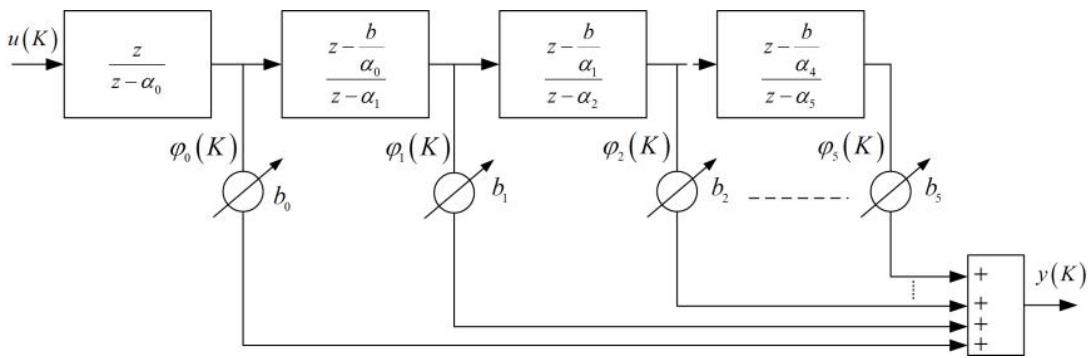

Figure 9

Block diagram of an adjustable model with the proposed orthogonal digital filter

The desired model of DPCM linear part is obtained by adjusting the following parameters: orthogonal filter poles $\alpha_{k}(k=0,1, \ldots, 5)$, coefficients $b_{k}(k=0,1, \ldots, 5)$, and the mapping parameter $b$. In the case of modeling a particular unknown system, parameters of the model should be adjusted in such a way that the model 
in Figure 9 corresponds as closely as possible to the unknown system. The process of modeling is performed in the well-known manner by introducing the same input to the system itself, as well as to its adjustable model based on the new cascade orthogonal digital filter (Figure 10) [15, 17, 18, 20].

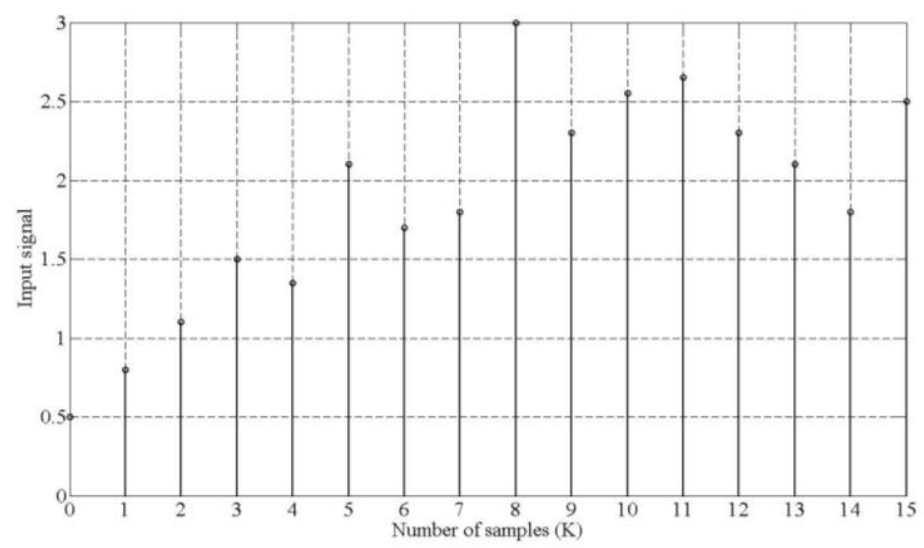

Figure 10

The input of DPCM linear part and the adjustable model

The next step is measuring the outputs from the system $y_{S}(t)$ and the filter $y_{M}(t)$, and calculating the mean squared error (criteria function) as in the previous experiment: $J=\frac{1}{N} \sum_{K=0}^{N}\left(y_{S}(K)-y_{M}(K)\right)^{2}$. Unknown parameters are obtained by minimization of $J$.

The genetic algorithm used in the experiment has same values for initial population, the number of generations like in previous one, and reproduction with six elite individuals. Also, we used the stochastic uniform selection and Gaussian mutation with shrinking. The used structure of chromosome was with 12 parameters coded by real numbers: $\alpha_{0}, \alpha_{1}, \alpha_{2}, \alpha_{3}, \alpha_{4}, \alpha_{5}, b_{0}, b_{1}, b_{2}, b_{3}, b_{4}$ and $b_{5}$.

The original signal (output from DPCM linear part) and the signal obtained using the adjustable model based on the orthogonal digital filter are given in Figure 11. 


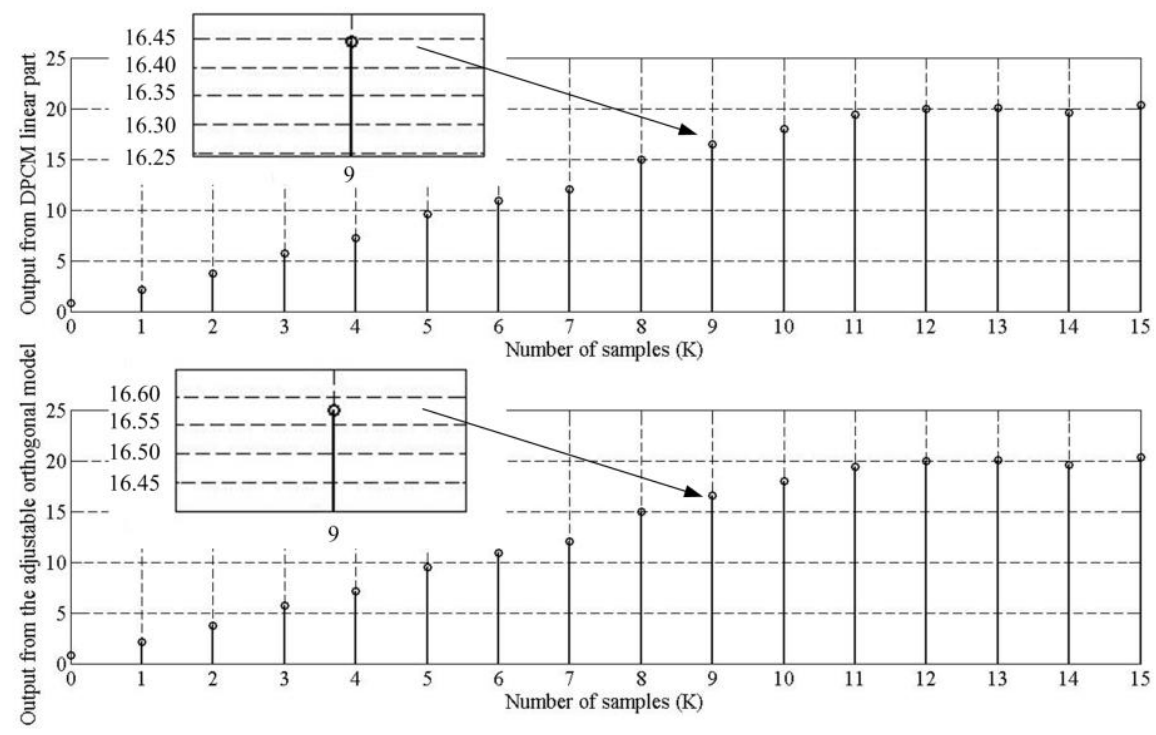

Figure 11

Outputs from DPCM linear part and the adjustable model

The results obtained for the optimal values of the parameters of the adjustable model are presented in Table 2 . The obtained value for the mean squared error is: $J_{\text {min }}=13.182 \cdot 10^{-3}$.

Table 2

Obtained parameters of the orthogonal digital model

\begin{tabular}{|l|l|l|l|l|l|l|}
\hline $\mathrm{k}$ & 0 & 1 & 2 & 3 & 4 & 5 \\
\hline$\alpha_{k}$ & 0.89880 & 0.80415 & 0.95560 & 0.71041 & -0.16135 & 0.86723 \\
\hline$b_{k}$ & 0.80535 & 0.55137 & 0.30523 & 0.05137 & -0.10907 & 0.02849 \\
\hline
\end{tabular}

We can notice a high level of matching between signals from DPCM linear part and the proposed orthogonal digital filter from the Figure 11.

Now, we have the model of DPCM linear part in the following form:

$$
W_{M}(z)=\sum_{k=0}^{5} b_{k} \prod_{i=0}^{k} \frac{z-\alpha_{i-1}^{*}}{z-\alpha_{i}}, \alpha_{-1}^{*}=0,
$$

where $\alpha_{k}^{*}=0.82 / \alpha_{k}$ and appropriate values of parameters are given in Table 2 . 


\section{Conclusion}

This paper presents a new class of cascade orthogonal filters based on the special inner product. A new method is applied in obtaining orthogonal Müntz polynomial from Malmquist rational functions and generalised Malmquist functions. Müntz polynomials obtained in this way are orthogonal with respect to the new inner product. Generalised Malmquist orthogonal functions were used for design of two new classes of orthogonal filters, for continuous (analogue) and discrete systems (digital filters). Müntz polynomials are used to prove orthogonality in the time domain, as well as to determine the outputs of these filters.

New filters are in the complex domain orthogonal on the contour which surrounds all the poles of the filters, while all zeroes lie outside this contour. Outputs of the filter are orthogonal with respect to the new inner product. Both analogue and digital filter were realized in our laboratory. Great matching between outputs from these filters and outputs obtained mathematically, by using Müntz polynomial, is shown.

The effectiveness of new classes of cascade orthogonal filters, analogue and digital, is demonstrated in the cases of determining a model of complex technological process in the tyre industry and for modeling the linear part of DPCM system, respectively. These filters can be applied in case of modeling dynamical systems when we adopt different mean squared errors (criteria functions) between the output of the process being modeled and the output from the adjustable filter (e.g. when the mean squared error is given in $\mathrm{dB}$ ).

The class of these filters with complex poles (both analogue and digital) can be also a subject for consideration in some future works.

\section{Acknowledgement}

This work was supported by the Ministry of Education, Science and Technological development of the Republic of Serbia (projects III 43007, III 44006 and TR 35005).

\section{References}

[1] N. Akhiezer, Theory of Approximation, New York, Dover Publications, 1956

[2] M. M. Djrbashian: Orthogonal Systems of Rational Functions on the Circle with Given Set of Poles, Dokl. Akad. Nauk SSSR, Vol. 147, pp. 12781281,1962

[3] M. M. Djrbashian: Orthogonal Systems of Rational Functions on the Circle, Akad. Nauk Armyan. SSR, Vol. 1, pp. 3-24, 106-125, 1996

[4] G. Szegö, Orthogonal Polynomials, American Mathematical Society, Colloquium Publications, 23, Providence, 1975 
[5] G. V. Badalyan: Generalisation of Legendre Polynomials and Some of Their Applications, Akad. Nauk. Armyan. SSR Izv. Ser. Fiz.-Mat. Estest. Tekhn. Nauk, Vol. 8, No. 5, pp. 1-28, 1955

[6] A. K. Taslakyan: Some Properties of Legendre Quasi-polynomials with Respect to a Müntz System, Mathematics, Erevan University, Erevan, Vol. 2, pp. 179-189, 1984

[7] G. Mastroianni and G. Milovanović, Interpolation Processes - Basic Theory and Applications, Springer-Verlag, Berlin, Heidelberg, 2008

[8] P. B. Borwein, T. Erdelyi, and J. Zhang: Müntz Systems and Orthogonal Müntz-Legendre Polynomials, Trans. Amer. Math. Soc. Vol. 342, No. 2, pp. 523-542, 1994

[9] M. M. Djrbashian: A Survey on the Theory of Orthogonal Systems and Some Open Problems, In P. Nevai (Ed.), Orthogonal Polynomials, pp. 135146, Springer, Netherlands, 1990

[10] K. Müller and A. Bultheel: Translation of the Russian paper "Orthogonal Systems of Rational Functions on the Circle", Report TW253, Katholieke Univ. Leuven, Leuven, 1997

[11] P. C. McCarthy, J. E. Sayre, and B. L. R. Shawyer: Generalized Legendre Polynomials, Journal of Mathematical Analysis and Applications, Vol. 177, No. 2, pp. 530-537, 1993

[12] B. Danković, G. V. Milovanović, and S. Rančić: Malmquist and Müntz Orthogonal Systems and Applications, in Inner Product Spaces and Applications, T. M. Rassias, eds., Addison-Wesley Longman, Harlow, pp. 22-41, 1997

[13] G. V. Milovanović, B. Danković, and S. Rančić: Some Müntz Orthogonal Systems, Journal of Computational and Applied Mathematics, Vol. 99, No. 1-2, pp. 299-310, 1998

[14] S. B. Marinković, B. Danković, M. S. Stanković, and P. M. Rajković: Orthogonality of Some Sequences of the Rational Functions and the Müntz Polynomials, Journal of Computational and Applied Mathematics, Vol. 163, No. 2, pp. 419-427, 2004

[15] D. Antić, Z. Jovanović, V. Nikolić, M. Milojković, S. Nikolić, and N. Danković: Modeling of Cascade-connected Systems using Quasiorthogonal Functions, Electronics and Electrical Engineering, Vol. 18, No. 10 , pp. 3-8, 2012

[16] B. Danković, S. Nikolić, M. Milojković, and Z. Jovanović: A Class of Almost Orthogonal Filters, Journal of Circuits, Systems, and Computer, Vol. 18, No. 5, pp. 923-931, 2009

[17] M. Milojković, D. Antić, M. Milovanović, S. S. Nikolić, S. Perić, and M. Almawlawe: Modeling of Dynamic Systems using Orthogonal Endocrine 
Adaptive Neuro-fuzzy Inference Systems, Journal of Dynamic Systems, Measurement, and Control, Vol. 137, No. 9, pp. DS-15-1098, 2015

[18] D. Antić, B. Danković, S. Nikolić, M. Milojković, and Z. Jovanović: Approximation Based on Orthogonal and Almost Orthogonal Functions, Journal of the Franklin Institute, Vol. 349, No. 1, pp. 323-336, 2012

[19] M. Milojković, S. Nikolić, B. Danković, D. Antić, and Z. Jovanović: Modelling of Dynamical Systems based on Almost Orthogonal Polynomials, Mathematical and Computer Modelling of Dynamical Systems, Vol. 16, No. 2, pp. 133-144, 2010

[20] D. Antić, S. Nikolić, M. Milojković, N. Danković, Z. Jovanović, and S. Perić: Sensitivity Analysis of Imperfect Systems using Almost Orthogonal Filters, Acta Polytechnica Hungarica, Vol. 8, No. 6, pp. 79-94, 2011

[21] S. Nikolić, D. Antić, B. Danković, M. Milojković, Z. Jovanović, and S. Perić: Orthogonal Functions Applied in Antenna Positioning, Advances in Electrical and Computer Engineering, Vol. 10, No. 4, pp. 35-42, 2010

[22] S. S. Nikolić, D. S. Antić, S. L. Perić, N. B. Danković, and M. T. Milojković: Design of Generalised Orthogonal Filters: Application to the Modelling of Dynamical Systems, International Journal of Electronics, Vol. 103, No. 2, pp. 269-280, 2016

[23] P. Heuberger, P. Van den Hof, and B. Wahlberg, Modelling and Identification with Rational Orthogonal Basis Functions, Springer-Verlag, London, 2005

[24] D. Trajković, V. Nikolić, D. Antić, and B. Danković: Analyzing, Modeling and Simulation of the Cascade Connected Transporters in Tire Industry using Signal and Bond Graphs, Machine Dynamics Problems, Vol. 29, No. 3, pp. 91-106, 2005

[25] N. S. Jayant and P. Noll, Digital Coding of Waveforms, Principles and Applications to Speech and Video, Chapter 6, Prentice-Hall, Englewood Cliffs NJ, USA, 1984

[26] N. B. Danković and Z. H. Perić: A Probability of Stability Estimation of DPCM System with the First Order Predictor, Facta Universitatis, Series: Automatic Control and Robotics, Vol. 12, No. 2, pp. 131-138, 2013

[27] N. Danković, Z. Perić, D. Antić, D. Mitić, and M. Spasić: On the Sensitivity of the Recursive Filter with Arbitrary Order Predictor in DPCM System, Serbian Journal of Electrical Engineering, Vol. 11, No. 4, pp. 609616, 2014 